\title{
AUTOMATIC KAPPA ANGLE ESTIMATION FOR AIR PHOTOS BASED ON PHASE ONLY CORRELATION
}

\author{
Z. Xiong *, D. Stanley, Y. Xin \\ PCI Geomatics Inc. 90 Allstate Parkway, Suite 501, Markham, ON L3R 6H3, Canada - (xiong, stanley, \\ xin)@pcigeomatics.com
}

Commission III, WG III/1

KEY WORDS: Kappa, Phase-Only Matching, Azimuth, Strip, Image Space, Object Space

\begin{abstract}
:
The approximate value of exterior orientation parameters is needed for air photo bundle adjustment. Usually the air borne GPS/IMU can provide the initial value for the camera position and attitude angle. However, in some cases, the camera's attitude angle is not available due to lack of IMU or other reasons. In this case, the kappa angle needs to be estimated for each photo before bundle adjustment. The kappa angle can be obtained from the Ground Control Points (GCPs) in the photo. Unfortunately it is not the case that enough GCPs are always available. In order to overcome this problem, an algorithm is developed to automatically estimate the kappa angle for air photos based on phase only correlation technique. This function has been embedded in PCI software. Extensive experiments show that this algorithm is fast, reliable, and stable.
\end{abstract}

\section{INTRODUCTION}

The initial approximations of photo's exterior orientation parameters (EOPs) are essential for air photo bundle adjustment. Without it, the bundle adjustment won't converge. Usually the air borne GPS/IMU can provide these value. However, in some cases, only the GPS data is available, and the camera's attitude angles are not available. For instance, the images are rotated during the preprocessing like lens distortion correction, or the IMU is not available when acquiring photos. In such situations, the attitude angles of the photos need to be estimated before bundle adjustment.

If a good distribution and quality of GCPs exist, the attitude angles can be easily computed [Dewitt, 1996]. Unfortunately this condition is not always met in practice. The alternative to estimate the initial value of exterior orientation parameters could be based on enough tie points and a number of GCPs. However, without the assistance of camera model, to collect enough quality tie points will be time consuming. For a commercial software, speed is always a key factor to its competitiveness. To overcome this challenge, we developed a new algorithm to automatically determine the kappa angle for air photos based on phase only correlation technique. Considering that the omega and phi angles can be set to zero for standard vertical photograph, if the kappa angle can be successfully estimated, then we have all initial values for the photo's exterior orientation parameters.

In the rest of this paper, we introduce the algorithm first. Then present the experiment and result. And finally give the conclusion.

\section{ALGORITHM}

The kappa angle of a photo is the angle from the north direction of the photo to the direction of true north at the location of the photo. The true north direction can be determined by air borne GPS data. If the north direction of the photo can be determined, then we get it.

We know that the air photos for mapping are acquired with side lapping and over lapping. From the GPS positions of adjacent photos, the azimuth of the flight line can be computed. This flight line azimuth directly tells us the true north at the location of the photo. If the flight line in the object space is projected to the image space, and the azimuth of the flight line can be determined in the image space, then the north direction of the photo can be determined.

Fortunately the phase only correlation can be used to determine the translation, rotation, and scaling of images (Castro, 1987; Chen, 1994; Li, 2006; Li, 2010; Lin, 2012; $\mathrm{He}, 2014)$. So the relative position of adjacent photos can be determined. With this information, the flight line azimuth in image space can be computed, and thus we get the north direction of the photo.

\subsection{Phase Only Correlation}

Phase only correlation was initially used to determine the translation of images (Chen, 1994; Li, 2006; Zheng, 2006). Suppose $f_{1}(x, y)$ and $f_{2}(x, y)$ are two images with translation relationship.

$f_{2}(x, y)=f_{1}\left(x-x_{0}, y-y_{0}\right)$

According to Fourier translation property, transforms of $f_{1}(x, y)$ and $f_{2}(x, y)$ are related by

$F_{2}(\xi, \eta)=e^{-j 2 \pi\left(\xi x_{0}+\eta y_{0}\right)} \times F_{1}(\xi, \eta)$

Where $F_{1}(\xi, \eta)$ and $F_{2}(\xi, \eta)$ are Fourier transforms of $f_{1}(x, y)$ and $f_{2}(x, y)$ individually. The cross-power spectrum of two images $f_{1}(x, y)$ and $f_{2}(x, y)$ with Fourier transforms $F_{1}(\xi, \eta)$ and $F_{2}(\xi, \eta)$ is defined as following. 
$\frac{F_{1}^{*}(\xi, \eta) F_{2}(\xi, \eta)}{\left|F_{1}^{*}(\xi, \eta) F_{2}(\xi, \eta)\right|}=e^{-j 2 \pi\left(\xi x_{0}+\eta y_{0}\right)}$

Where $F_{1}^{*}(\xi, \eta)$ is the complex conjugate of $F_{1}(\xi, \eta)$. The inverse Fourier transform of $e^{-j 2 \pi\left(\xi x_{0}+\eta y_{0}\right)}$ is an impulse; that means the value of such function is approximately zero everywhere except at the displacement of two images (Reddy, 1996).

The phase only correlation is also used to determine the scale and rotation angle of images (Reddy, 1996; Zheng, 2006; Sarvaiya, 2012). It is the same as above that suppose $f_{1}(x, y)$ and $f_{2}(x, y)$ are two images with scaling and rotation relationship.

$f_{2}(x, y)=f_{1}\left(\sigma^{-1}\left(x \cos \theta_{0}+y \sin \theta_{0}\right), \sigma^{-1}\left(-x \sin \theta_{0}+\right.\right.$ $\left.y \cos \theta_{0}\right)$

$f_{1}(x, y)$ and $f_{2}(x, y)$ can be expressed as following in their polar representation.

$f_{2 \rho}=f_{1 \rho}\left(\rho / \sigma, \theta-\theta_{0}\right)$

By converting the $\rho$ axes to logarithmic scale, scaling can be reduced to a translation movement.

$f_{2 \rho}=f_{1 \rho}\left(\lambda-\lambda_{0}, \theta-\theta_{0}\right)$

Where, $\lambda=\log \rho, \lambda_{0}=\log \sigma$.

Obviously the rotation and scaling are converted to translation in the polar coordinate system. According to Fourier translation property, by applying phase only correlation to the images in polar representation, the scale and rotation angle can be determined.

\subsection{Estimation of Kappa Angle}

The kappa angle can be calculated using the azimuth angle of flight line in both image space and object space. And the azimuth angle in object space can be obtained from the air borne GPS data. Therefore, the key point to determine a photo's kappa angle is the azimuth angle of the flight line in image space. In this paper, an algorithm to estimate kappa angles for air photos based on phase matching is introduced. This method can determine the translation, rotation angle, and scale between images and thus determine the azimuth angle of flight line in image space.

Suppose we have two photos $\mathrm{a}$ and $\mathrm{b}$ as following (Figure 1). From the air borne GPS data, we can compute the azimuth of flight line. Although the phase center of the GPS antenna is probably different to the exposure station, the direction of the flight line based on GPS data is still consistent with the direction from exposure $\mathrm{O}_{1}$ to exposure $\mathrm{O}_{2}$. Thus we can consider $\beta$ as the azimuth of flight line.

By applying phase matching on the adjacent photos, the translation, rotation angle, and scale can be determined. From image a to image $b$, following transformation can be obtained.

$x^{\prime}=\sigma^{-1}\left(x \cos \theta_{0}+y \sin \theta_{0}+x_{0}\right)$

$y^{\prime}=\sigma^{-1}\left(-x \sin \theta_{0}+y \cos \theta_{0}+y_{0}\right)$

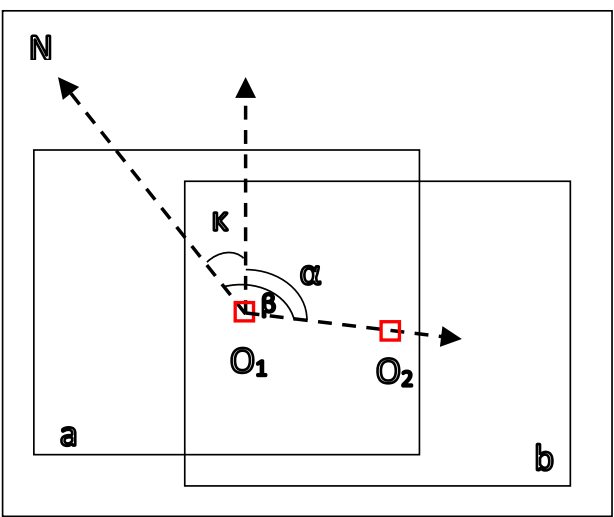

Figure 1. Kappa angle

The coordinates of $\mathrm{O}_{2}$ in image $\mathrm{b}$ can be transformed into coordinates in image a according to the above equations. Then, in the image space, the azimuth angle $\alpha$ of the line of two photo centers in image a can be computed. With the azimuth angle of flight line in image space $\alpha$ and azimuth angle of flight line in the object space ${ }_{\beta}$, the kappa angle of image a can be obtained.

$\kappa=\alpha-\beta$

\subsubsection{Group Photos Into Strips}

In a block of air photos, each photo is connected with its neighboring photos by over lapping and side lapping. Any photo which has no connection with other photos needs to be removed from the block. The remaining photos of the block need to be grouped into strips. The reasons include (1) the azimuth of flight line for the last photo of a strip cannot be directly computed using the GPS data; (2) we assume that the photos are acquired in one flight, that means the camera is mounted on the platform without rotation or the images are not rotated relatively to each other in a strip; (3) we assume that, in a strip, the azimuth of flight line in image space for each photo is approximately equal. So we can use strip average azimuth of flight line in image space to replace photo azimuth of flight line in image space, because the phase matching may fail sometime.

To group photos into strips can be based on following criteria.

(1) The difference of azimuth angle. For two neighboring photos, if the difference of azimuth angles is greater than a threshold, like thirty degree, we can consider these two photos belong to different strips.

(2) Time gap. Each GPS data with a photo contains the time of the exposure of the photo. If the time difference of two neighboring photos is greater than a threshold, these two neighboring photos can be considered belong to different strips.

(3) Distance between neighboring photos. If the distance between two neighboring photos is greater than a threshold, these two photos can be considered belong to different strips. 


\subsubsection{Compute Azimuth Of Flight Line}

For each photo, the approximate location of camera's exposure station can be obtained from GPS data. So we can calculate the azimuth of flight line for each photo (Figure 2). For the last photo of each strip, we directly assign the same azimuth of its former photo. For instance, a strip consists of two photos a and b (Figure 2). The azimuth of photo a can be computed using GPS data as $\beta$. The photo $b$ is the last photo in the strip, and its azimuth is assigned the same azimuth of its former photo a. So the azimuth of photo $b$ is assigned $\beta$.

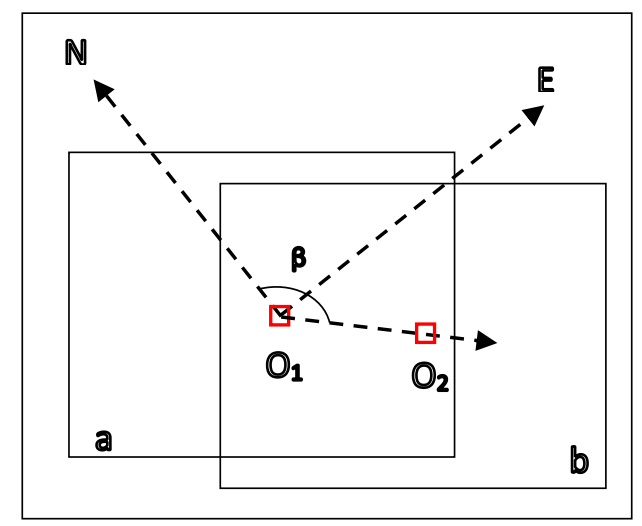

Figure 2. Azimuth of flight line

\subsubsection{Phase Matching}

By applying phase matching to two neighboring photos, the translation, rotation, and scale can be recovered. This procedure consists of two steps (Reddy, 1996; Sarvaiya, 2012):

(1) Transform both images to frequency domain by Fourier transform, then resample the $\mathrm{N}$ by $\mathrm{N}$ spectral magnitude onto an $\mathrm{M}$ by K polar-log grid as following by log-polar transform.

$$
x(m, k)=\frac{\frac{N}{2}-1}{M-1}(M-1)^{m /(M-1)} \cos \left(\frac{\pi k}{K}\right)+\frac{N}{2}
$$

$$
y(m, k)=\frac{\frac{N}{2}-1}{M-1}(M-1)^{m /(M-1)} \sin \left(\frac{\pi k}{K}\right)+\frac{N}{2}
$$

Where $\mathrm{m}$ is the discrete radial coordinate and $\mathrm{k}$ the discrete angular coordinate, and $\mathrm{x}$ and $\mathrm{y}$ are the Cartesian frequency coordinates.

In the log-polar domain, apply the phase only correlation to recover the scale and rotation.

(2) Once the scale and rotation angle are obtained, the image with the higher resolution is scaled and rotated. Then apply phase matching to recover the translation.

In order to reduce the edge effect, a high-pass filter is applied in the frequency domain (Sarvaiya, 2012).

\subsubsection{Compute Azimuth Of Flight Line In Image Space}

Once the rotation angle, scale and translation are recovered, the image coordinates of the central point of the second photo can be transformed into the image coordinates in the first image coordinate system (7). Then the azimuth of flight line in image domain $\boldsymbol{\alpha}$ can be calculated.

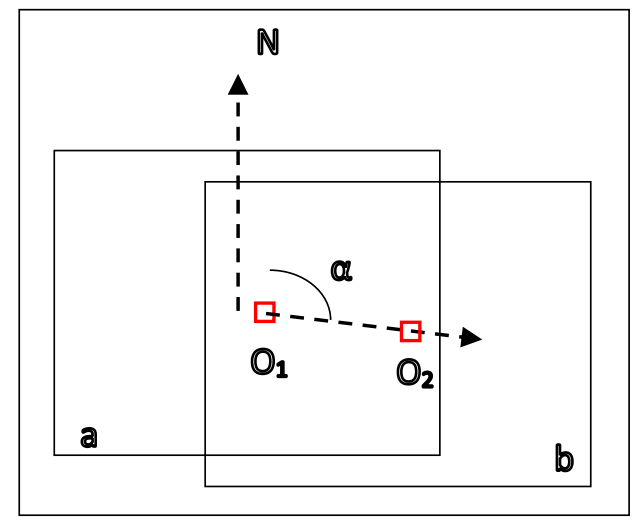

Figure 3. Azimuth of flight line in image space

Although each photo in a strip can be assigned an azimuth of flight line, these azimuths don't have significant difference, because the photos with quite different azimuth have been grouped to different strips. Therefore, once a phase matching between two photos fails and the scale, rotation angle, and translation cannot be recovered, and thus the azimuth of flight line in image space cannot be determined, the average azimuth in a strip can be assigned to those photos.

\subsubsection{Compute Kappa Angle}

After the azimuth of flight line in image space and object space for each photo are obtained, the kappa angle for each photo can be calculated as (8).

\section{EXPERIMENT}

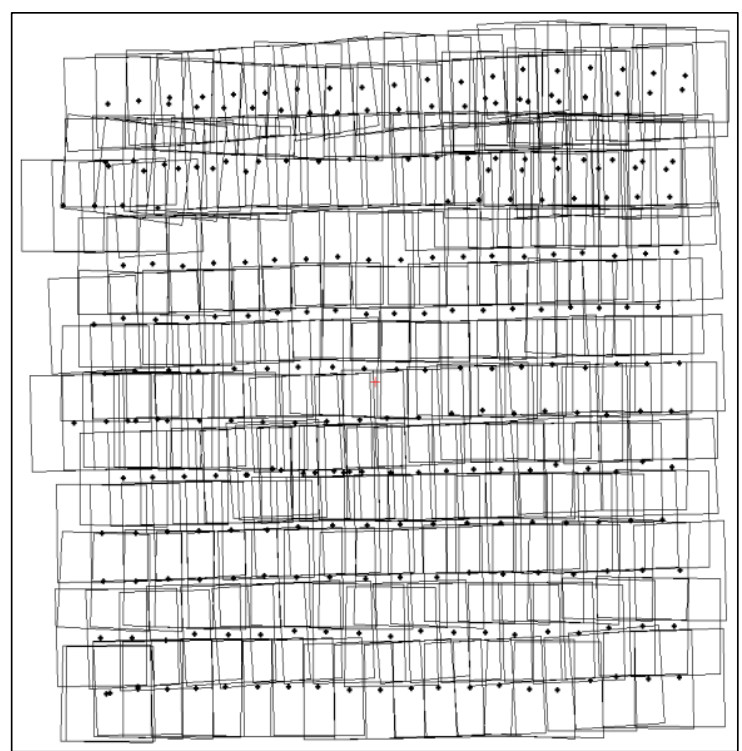

Figure 4. A Block of Air Photos 
This algorithm has been embedded in PCI software. We tested this function extensively with air photos. Here we just demonstrate this algorithm by one example. In this project, we have a block of 266 air photos which were acquired approximately vertically by a UAV as figure 4 .

GPS data for each photo is available. By applying phase matching, kappa angle was recovered for each photo (Figure 5). The phase matching of 266 images took only several seconds in our desktop machine.

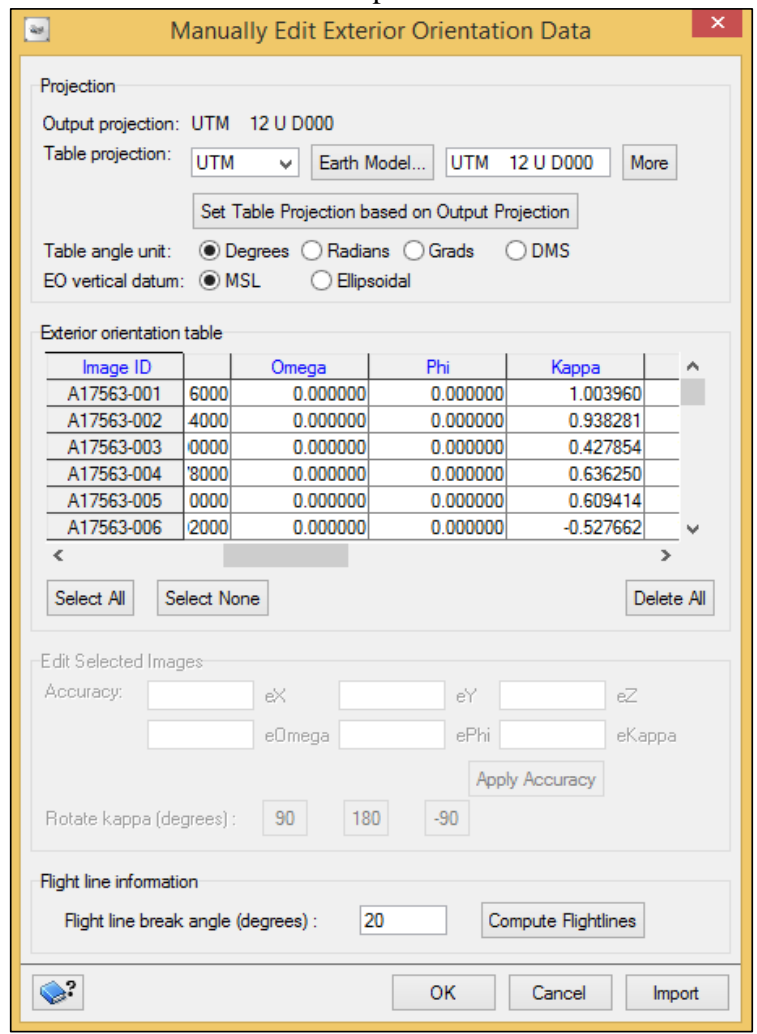

Figure 5. Recovered Kappa angle

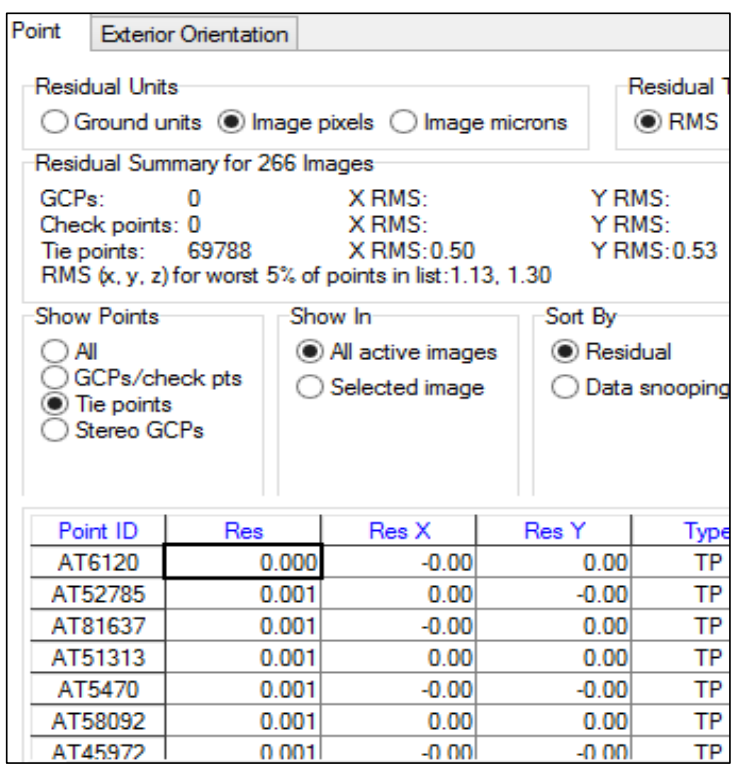

Figure 6. Bundle adjustment result
All photos are acquired vertically. We set omega and phi to zero for each photo. Thus the approximation values of all six exterior orientation parameters for each photo are obtained. Based on this approximate exterior orientation parameters, 69788 tie points were collected. After bundle adjustment, an accuracy of 0.5 pixels for RMS $\mathrm{x}$ and 0.53 pixels for RMS y was obtained (Figure 6).

\section{CONCLUSIONS}

In aerial triangulation, the approximation of exterior orientation parameters (EOPs) are critical to bundle adjustment. For vertical air photos, if only the three linear EOPs are available and the other non-linear EOPs are unknowns, the kappa angles need to be estimated. Here the algorithm of kappa angle estimation based on the phase only correlation has been presented. It is extensively tested and found to be successful for all standard air photos when the air borne GPS data is available. Experiments show that this algorithm is fast, reliable, stable, and can always give satisfactory estimation for kappa angle.

\section{REFERENCES}

Castro, E., Morandi, C., 1987. Registration of translated and rotated images using Finite Fourier transforms. IEEE Transactions On Pattern Analysis and Machine Intelligence, Vol. PAMI-9, No. 5. Pp 700-703.

Chen, Q., Defrise, M., and Deconink, F., 1994. Symmetric phase-only matched filtering of Fourier-Mellin transforms for image registration and recognition. IEEE Transactions On Pattern Analysis and Machine Intelligence, Vol. 16, No. 12. Pp 1156-1168.

Dewitt, B., 1996. Initial approximations for the threedimensional conformal coordinate transformation. Photogrammetric Engineering \& Remote Sensing, Vol. 62, No. 1. Pp79-83.

He, H., Zhang, Y., Huang, S., 2014. Phase correlation supported low altitude images matching with repeated texture. Geomatics and Information Science of Wuhan University. Vol. 39, No. 10. Pp 1204-1207.

Li, R., Wang, J., Guo, F., Du, J., 2010. Automatic registration method for different temporal remote sensing images on improved Fourier-Mellin algorithm. Computer Engineering and Applications. Vol. 46, No. 16. Pp178181.

Li, X., Zhao, X., Zheng, L., Hu, Z., 2006. An image registration technique based on Fourier-Mellin transform and its extended applications. Chinese Journal of Computers. Vol. 29, No. 3. Pp466-472.

Lin, H., Liang, L., Du, P., Sun, H., 2012. Image registration based on Fourier-Mellin transform. Geomatics and Information Science of Wuhan University. Vol. 37, No. 6. Pp 649-652.

Reddy, B., Chatterji, B., 1996. An FFT-based technique for translation, rotation, and scale-invariant image registration. IEEE Transactions On Image Processing. Vol. 5, No. 8. Pp1266-1271. 
Sarvaiya, J., Patnaik, S., Kothari, K., 2012. Image registration using Log Polar transform and phase correlation to recover higher scale. Journal of Pattern Recognition Research. Vol. 2012, No. 7. Pp90-105.

Zheng, Z., Ye, Z., 2006. Image registration algorithm based on phase-correlation. Journal of Data Acquisition \& Processing. Vol. 21, No. 4. Pp444-449.

Revised March 2016 